\title{
Distributed algorithm for target localization in wireless sensor networks using RSS and AoA measurements
}

Article in Pervasive and Mobile Computing · October 2016

DOI: 10.1016/j.pmcj.2016.09.013

CITATIONS

13

4 authors, including:

Slavisa Tomic

New University of Lisbon

31 PUBLICATIONS 187 CITATIONS

SEE PROFILE

Paulo Carvalho

Institute for the Development of New Technolo...

133 PUBLICATIONS 483 CITATIONS

SEE PROFILE
READS

33

Rui Dinis

New University of Lisbon

455 PUBLICATIONS 2,984 CITATIONS

SEE PROFILE

Some of the authors of this publication are also working on these related projects: 


\title{
Distributed Algorithm for Target Localization in Wireless Sensor Networks Using RSS and AoA Measurements
}

\author{
Slavisa Tomic ${ }^{\mathrm{a}, *}$, Marko Beko ${ }^{\mathrm{b}, \mathrm{c}}$, Rui Dinis ${ }^{\mathrm{d}, \mathrm{e}}$, Paulo Montezuma ${ }^{\mathrm{d}, \mathrm{e}}$ \\ ${ }^{a}$ ISR/IST, LARSyS, Universidade de Lisboa, 1049-001 Lisbon, Portugal \\ ${ }^{b}$ Universidade Lusófona de Humanidades e Tecnologias, Campo Grande 376, 1749 - 024 \\ Lisboa, Portugal \\ ${ }^{c}$ CTS/UNINOVA, Campus da FCT/UNL, Monte de Caparica, 2829-516 Caparica, Portugal \\ ${ }^{d}$ Instituto de Telecomunicações, Av. Rovisco Pais 1, Torre Norte, piso 10, 1049 - 001 \\ Lisboa, Portugal \\ ${ }^{e}$ Dep. ${ }^{\circ}$ de Eng. ${ }^{a}$ Electrotécnica, FCT/UNL, 2829-516 Caparica, Portugal
}

\begin{abstract}
This paper addresses target localization problem in a cooperative 3-D wireless sensor network (WSN). We employ a hybrid system that fuses distance and angle measurements, extracted from the received signal strength (RSS) and angle-ofarrival (AoA) information, respectively. Based on range measurement model and simple geometry, we derive a novel non-convex estimator based on the least squares (LS) criterion. The derived non-convex estimator tightly approximates the maximum likelihood (ML) one for small noise levels. We show that the developed non-convex estimator is suitable for distributed implementation, and that it can be transformed into a convex one by applying a second-order cone programming (SOCP) relaxation technique. We also show that the developed non-convex estimator can be transformed into a generalized trust region subproblem (GTRS) framework, by following the squared range (SR) approach. The proposed SOCP algorithm for known transmit powers is then generalized to the case where the transmit powers are different and not known. Furthermore, we provide a detailed analysis of the computational complexity of the proposed
\end{abstract}

\footnotetext{
${ }^{*}$ Corresponding author

Email addresses: stomic@isr.ist.utl.pt (Slavisa Tomic), beko.marko@ulusofona.pt, mbeko@uninova.pt (Marko Beko), rdinis@fct.unl.pt (Rui Dinis), pmc@uninova.pt (Paulo Montezuma)
}

Preprint submitted to Journal of ${ }^{A} T_{E} X$ Templates

August 5, 2016 
algorithms. Our simulation results show that the new estimators have excellent performance in terms of the estimation accuracy and convergence, and they confirm the effectiveness of combining two radio measurements.

Keywords: Wireless localization, wireless sensor network (WSN), received signal strength (RSS), angle-of-arrival (AoA), second-order cone programming (SOCP), generalized trust region sub-problem (GTRS).

\section{Introduction}

In recent years wireless sensor networks (WSNs) have been used in various areas, like event detection (fires, floods) [1], monitoring (health care, industrial, agricultural, environmental) [2, 3], energy-efficient routing [4], exploration (un-

5 derground, deep water, outer space) [5], and surveillance [6], to name a few. A key element in many practical applications is to accurately determine the locations of sensors $[7,8]$, namely in search and rescue missions or to enhance the network coverage. Although global positioning system (GPS) receivers can be used to locate the sensors, GPS is ineffective in indoor, dense urban and forest environments or canyons [9]. Besides, installing a GPS receiver in each sensor would be extremely expensive in large-scale WSNs, which would restrict its applicability $[8,10]$. Hence, development of localization strategies from different terrestrial radio frequency $(\mathrm{RF})$ sources is of great practical interest.

Nowadays, RF signals come from a wide variety of sources and technologies, and they can be used for localization purpose. In a WSN, the locations of the unknown sensors (targets) are determined by using a kind of localization schemes that typically rely on the locations of the reference sensors (anchors) and range measurements between them. Range measurements can be extracted from different characteristics of the radio signal, such as time-of-arrival (ToA) [11], 20 time-difference-of-arrival (TDoA) [12], round-trip time (RTT), time of flight (ToF) [13], angle-of-arrival (AoA) [14] or received signal strength (RSS) [15, 16], depending on the available hardware. Recently, hybrid systems that fuse two measurements of the radio signal have been investigated [17]-[27]. Hybrid sys- 
tems profit by exploiting the benefits of combined measurements (more available information), taking advantage of the strongest points of each technique and minimizing their drawbacks. On the other hand, the price to pay for using such systems is the increased complexity of network devices, which increases the network implementation costs $[8,10]$.

Typically, data processing in localization schemes can be performed in a centralized or a distributed manner [28]. On the one hand, existence of a central processor (sensor or a base station) is required for the former approach. Central processor gathers all measurements via wireless transmissions and produces a map of the entire network [20]-[23]. However, in large-scale networks, a high energy drain is likely to occur at and near the central processor, caused by a bot35 tlenecks [10]. Likewise, the computational complexity of a centralized approach depends highly on the network size. In many applications a central processor (or one with enough computational capacity) is not available. Furthermore, confidentiality may prevent sharing objective functions between sensors in some practical applications [29]. On the other hand, the later approach is distinguished by low computational complexity and high-scalability, which makes it a preferable solution for large-scale and highly-dense networks [28]. However, distributed algorithms are executed iteratively, which makes them vulnerable to error propagation and raises the energy consumption. In general, when the average number of hops to the central processor is higher than the necessary number of iterations required for convergence, the distributed approach is likely to be more energy-efficient [8].

Localization of a sensor network with small number of anchors using graph theory and binary data has drawn much attention recently [30]-[34]. In [35] a study of traditional non-cooperative RSS- and AoA-based localization meth50 ods for visible light communication systems was presented. The approaches in [17]-[19] are based on the fusion of RSS and ToA measurements. A hybrid system that merges range and angle measurements was investigated in [20]. The authors in [20] proposed two estimators to solve the non-cooperative target localization problem in a 3-D scenario: linear least squares (LS) and optimization 
based. The LS estimator is a relatively simple and well known estimator, while the optimization based estimator was solved by Davidson-Fletcher-Powell algorithm [36]. In [21], the authors derived an LS and a maximum likelihood (ML) estimator for a hybrid scheme that combines RSS difference (RSSD) and AoA measurements. Non-linear constrained optimization was used to estimate the target's location from multiple RSS and AoA measurements. Both LS and ML estimators in [21] are $\lambda$-dependent, where $\lambda$ is a non-negative weight assigned to regulate the contribution from RSS and AoA measurements. A selective weighted LS (WLS) estimator for RSS/AoA localization problem was proposed in [22]. The authors determined the target location by exploiting weighted ranges from the two nearest anchor measurements, which were combined with the serving base station AoA measurement. In [21] and [22], authors investigated the non-cooperative hybrid RSS/AoA localization problem for a 2-D scenario only. A WLS estimator for a 3-D RSSD/AoA non-cooperative localization problem when the transmit power is unknown was presented in [23]. However, the authors in [23] only investigated a small-scale WSN, with extremely low noise power. Two estimators for 3-D non-cooperative RSS/AoA localization problem based on convex optimization and squared-range approach were proposed in [24]. The work in [25] addressed an RSS/AoA non-cooperative localization problem in 2-D non-line of sight environments. The authors in [25] proposed 75 an alternating optimization algorithm, composed of fixing the value of the scatter orientation and solving the semidefinite programming (SDP) representation of the localization problem and later using the obtained location estimate to update the value of the scatter orientation, for localizing a mobile target in a WSN. In [26] a cooperative RSS/AoA localization problem was investigated.

80 The authors in [26] proposed an SDP estimator to simultaneously localize multiple targets. However, the proposed algorithm is for centralized applications only, and its computational complexity depends highly on the network size. Convex optimization techniques were employed in [27] to solve the cooperative RSS/AoA target localization problem with unknown transmit powers in a distributed manner. 
Apart from [26] and [27], all mentioned approaches investigate non-cooperative localization problem only, where the location of a single target, which communicates with anchors exclusively, is determined at a time. Contrary to these approaches, in this paper we investigate the target localization problem in a large-scale WSN, where the number of anchors is scarce and the communication range of all sensors is restricted (e.g., to prolong sensor's battery life). In such settings, only some targets can directly communicate with anchors; therefore, cooperation between any two sensors within the communication range is required in order to acquire sufficient amount of information to perform localization. We design novel distributed hybrid localization algorithms based on second-order cone programming (SOCP) relaxation and generalized trust region sub-problems (GTRS) framework that take advantage of combined RSS/AoA measurements with known transmit power to estimate the locations of all targets in a WSN. The proposed algorithms are distributed in the sense that no central sensor coordinates the network, all communications occur exclusively between two incident sensors and the data associated with each sensor are processed locally. First, the non-convex and computationally complex ML estimation problem is broken down into smaller sub-problems, i.e., the local ML estimation problem for each target is posed. By using the RSS propagation model and simple geometry, we derive a novel local non-convex estimator based on the LS criterion, which tightly approximates the local ML one for small noise levels. Then, we show that the derived non-convex estimator can be transformed into a convex SOCP estimator that can be solved efficiently by interior-point algorithms [37]. Furthermore, following the squared range (SR) approach, we propose a suboptimal SR-WLS estimator based on the GTRS framework, which can be solved exactly by a bisection procedure [38]. We then generalize the proposed SOCP estimator for known transmit powers to the case where the target transmit powers are different and not known.

Throughout the paper, upper-case bold type, lower-case bold type and regular type are used for matrices, vectors and scalars, respectively. $\mathbb{R}^{n}$ denotes the $n$-dimensional real Euclidean space. The operators $\otimes$ and $(\bullet)^{T}$ denote the 
Kronecker product and transpose, respectively. The normal (Gaussian) distribution with mean $\mu$ and variance $\sigma^{2}$ is denoted by $\mathcal{N}\left(\mu, \sigma^{2}\right) \cdot \operatorname{diag}(\boldsymbol{x})$ denotes a square diagonal matrix in which the elements of vector $\boldsymbol{x}$ form the main diagonal of the matrix, and the elements outside the main diagonal are zero. The $N$-dimensional identity matrix is denoted by $\boldsymbol{I}_{N}$ and the $M \times N$ matrix of all zeros by $\mathbf{0}_{M \times N}$ (if no ambiguity can occur, subscripts are omitted). $\|\boldsymbol{x}\|$ denotes the vector norm defined by $\|\boldsymbol{x}\|=\sqrt{\boldsymbol{x}^{T} \boldsymbol{x}}$, where $\boldsymbol{x} \in \mathbb{R}^{n}$ is a column vector.

The remainder of this work is organized as follows. In Section 2, the RSS and AoA measurement models are introduced and the target localization problem is formulated. Section 3 presents the development of the proposed distributed estimators. In Section 4 we provide an analysis about the computational complexity, while in Section 5 we discuss the performance of the proposed algorithms. Finally, Section 6 summarizes the main conclusions.

\section{Problem Formulation}

Consider a large-scale WSN with $M$ targets and $N$ anchors, randomly deployed over a region of interest. The considered network can be seen as a connected graph, $\mathcal{G}(\mathcal{V}, \mathcal{E})$, with $|\mathcal{V}|=M+N$ vertices and $|\mathcal{E}|$ edges, where $|\bullet|$ represents the cardinality (the number of elements in a set) of a set. The set of targets and the set of anchors are respectively labeled as $\mathcal{T}(|\mathcal{T}|=M)$ and $\mathcal{A}$ $(|\mathcal{A}|=N)$, and their locations are denoted by $\boldsymbol{x}_{1}, \boldsymbol{x}_{2}, \ldots, \boldsymbol{x}_{M}$ and $\boldsymbol{a}_{1}, \boldsymbol{a}_{2}, \ldots, \boldsymbol{a}_{N}$ $\left(\boldsymbol{x}_{i}, \boldsymbol{a}_{j} \in \mathbb{R}^{3}, \forall i \in \mathcal{T}\right.$ and $\left.\forall j \in \mathcal{A}\right)$, respectively. To save power (battery duration conditions the lifetime of a network), it is assumed that all sensors have limited communication range, $R$. Thus, two sensors, $i$ and $j$, can exchange information if and only if they are within the communication range of each other. The sets of all target/anchor and target/target connections (edges) are defined as $\mathcal{E}_{\mathcal{A}}=\left\{(i, j):\left\|\boldsymbol{x}_{i}-\boldsymbol{a}_{j}\right\| \leq R, \forall i \in \mathcal{T}, \forall j \in \mathcal{A}\right\}$ and $\mathcal{E}_{\mathcal{T}}=\left\{(i, k):\left\|\boldsymbol{x}_{i}-\boldsymbol{x}_{k}\right\| \leq R, \forall i, k \in \mathcal{T}, i \neq k\right\}$, respectively.

For ease of expression, let us define a matrix $\boldsymbol{X}=\left[\boldsymbol{x}_{1}, \boldsymbol{x}_{2}, \ldots, \boldsymbol{x}_{M}\right](\boldsymbol{X} \in$ 
locations by using a hybrid system that fuses range and angle measurements.

Throughout this work, it is assumed that the range measurements are obtained from the RSS information exclusively, since ranging based on RSS requires the lowest implementation costs [8]. The RSS between two sensors $i$ and $j$ which are within the communication range of each other (from the transmitting sensor $), P_{i j}(\mathrm{dBm})$, is modeled as:

$$
\begin{aligned}
& P_{i j}^{\mathcal{A}}=P_{0 i}-10 \gamma \log _{10} \frac{\left\|\boldsymbol{x}_{i}-\boldsymbol{a}_{j}\right\|}{d_{0}}+n_{i j}, \forall(i, j) \in \mathcal{E}_{\mathcal{A}}, \\
& P_{i k}^{\mathcal{T}}=P_{0 i}-10 \gamma \log _{10} \frac{\left\|\boldsymbol{x}_{i}-\boldsymbol{x}_{k}\right\|}{d_{0}}+n_{i k}, \forall(i, k) \in \mathcal{E}_{\mathcal{T}},
\end{aligned}
$$

(see $[39,40])$, where $P_{0 i}(\mathrm{dBm})$ denotes the reference power at a distance $d_{0}$ $\left(\left\|\boldsymbol{x}_{i}-\boldsymbol{a}_{j}\right\| \geq d_{0},\left\|\boldsymbol{x}_{i}-\boldsymbol{x}_{k}\right\| \geq d_{0}\right)$ from the transmitting sensor (which depends on the transmit power [10]), $\gamma$ is the path loss exponent (PLE) between sensors $i$ and $j$ which indicates the rate at which the power decreases with distance, and $n_{i j}$ and $n_{i k}$ are the log-normal shadowing terms modeled as $n_{i j} \sim \mathcal{N}\left(0, \sigma_{n_{i j}}^{2}\right)$, $n_{i k} \sim \mathcal{N}\left(0, \sigma_{n_{i k}}^{2}\right)$. We assume that the target/target RSS measurements are symmetric $^{1}$, i.e., $P_{i k}^{\mathcal{T}}=P_{k i}^{\mathcal{T}}, \forall(i, k) \in \mathcal{E}_{\mathcal{T}}, i \neq k$.

To obtain the AoA measurements (both azimuth and elevation angles), we assume that either antenna arrays or a directional antenna is implemented at anchors [20,41,42], or that the anchors are equipped with video cameras [43]. In order to make use of the AoA measurements from different anchors, the orientation information is required, which can be obtained by implementing a digital compass at each anchor [20, 41]. However, a digital compass introduces an error in the AoA measurements due to its static accuracy. For the sake of simplicity and without loss of generality, we model the angle measurement error and the orientation error as one random variable in the rest of this paper.

Fig. 1 gives an illustration of a target and an anchor locations in a 3-D space. As shown in Fig. $1, \boldsymbol{x}_{i}=\left[x_{i \mathrm{x}}, x_{i \mathrm{y}}, x_{i \mathrm{z}}\right]^{T}$ and $\boldsymbol{a}_{j}=\left[a_{j \mathrm{x}}, a_{j \mathrm{y}}, a_{j z}\right]^{T}$ are respectively

\footnotetext{
${ }^{1}$ This assumption is made without loss of generality; it is readily seen that, if $P_{i k}^{\mathcal{T}} \neq P_{k i}^{\mathcal{T}}$, then it is enough to replace $P_{i k}^{\mathcal{T}} \leftarrow\left(P_{i k}^{\mathcal{T}}+P_{k i}^{\mathcal{T}}\right) / 2$ and $P_{k i}^{\mathcal{T}} \leftarrow\left(P_{i k}^{\mathcal{T}}+P_{k i}^{\mathcal{T}}\right) / 2$ when solving the localization problem.
} 


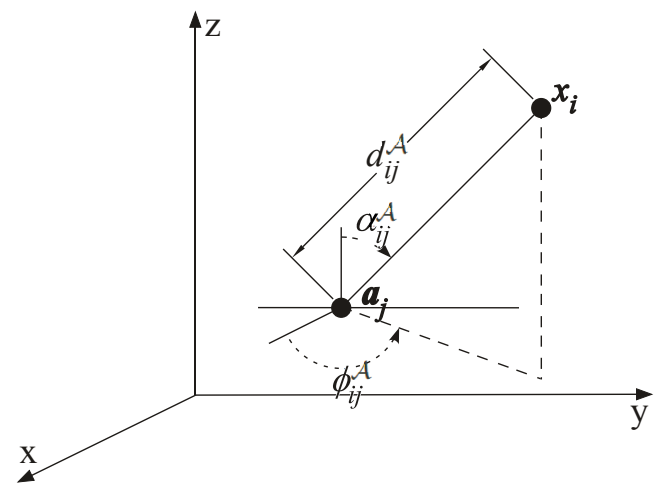

Figure 1: Illustration of a target and anchor locations in a 3-D space.

the unknown coordinates of the $i$-th target and the known coordinates of the $j$-th anchor, while $d_{i j}^{\mathcal{A}}, \phi_{i j}^{\mathcal{A}}$ and $\alpha_{i j}^{\mathcal{A}}$ represent the distance, azimuth angle and elevation angle between the $i$-th target and the $j$-th anchor, respectively. The ML estimate of the distance between two sensors can be obtained from the RSS measurement model (1) as follows [8]:

$$
\widehat{d}_{i j}= \begin{cases}d_{0} 10^{\frac{P_{0 i}-P_{i j}^{\mathcal{A}}}{10 \gamma}}, & \text { if } j \in \mathcal{A}, \\ d_{0} 10^{\frac{P_{0 i}-P_{i j}^{\top}}{10 \gamma}}, & \text { if } j \in \mathcal{T} .\end{cases}
$$

Applying simple geometry, azimuth and elevation angle measurements ${ }^{2}$ can be modeled respectively as [20]:

$$
\phi_{i j}^{\mathcal{A}}=\arctan \left(\frac{x_{i \mathrm{y}}-a_{j \mathrm{y}}}{x_{i \mathrm{x}}-a_{j \mathrm{x}}}\right)+m_{i j}, \text { for }(i, j) \in \mathcal{E}_{\mathcal{A}},
$$

and

$$
\alpha_{i j}^{\mathcal{A}}=\arccos \left(\frac{x_{i \mathrm{z}}-a_{j \mathrm{z}}}{\left\|\boldsymbol{x}_{i}-\boldsymbol{a}_{j}\right\|}\right)+v_{i j}, \text { for }(i, j) \in \mathcal{E}_{\mathcal{A}},
$$

where $m_{i j}$ and $v_{i j}$ are the measurement errors of azimuth and elevation angles, respectively, modeled as $m_{i j} \sim \mathcal{N}\left(0, \sigma_{m_{i j}}^{2}\right)$ and $v_{i j} \sim \mathcal{N}\left(0, \sigma_{v_{i j}}^{2}\right)$.

\footnotetext{
${ }^{2}$ Note that we consider here the case where only anchors have the necessary equipment to perform the respective angle measurements. An alternative approach would be to provide the necessary equipment to all sensors. However, our simulations showed that there is no gain for such a setting, and it would severely raise the overall network implementation costs.
} 
Given the observation vector $\boldsymbol{\theta}=\left[\boldsymbol{P}^{T}, \boldsymbol{\phi}^{T}, \boldsymbol{\alpha}^{T}\right]^{T}\left(\boldsymbol{\theta} \in \mathbb{R}^{3\left|\mathcal{E}_{\mathcal{A}}\right|+\left|\mathcal{E}_{\mathcal{T}}\right|}\right)$, where $\boldsymbol{P}=\left[P_{i j}^{\mathcal{A}}, P_{i k}^{\mathcal{T}}\right]^{T}, \boldsymbol{\phi}=\left[\phi_{i j}^{\mathcal{A}}\right]^{T}, \boldsymbol{\alpha}=\left[\alpha_{i j}^{\mathcal{A}}\right]^{T}$, the conditional probability density function $(\mathrm{PDF})$ is given as:

$$
p(\boldsymbol{\theta} \mid \boldsymbol{X})=\prod_{i=1}^{3\left|\mathcal{E}_{\mathcal{A}}\right|+\left|\mathcal{E}_{\mathcal{T}}\right|} \frac{1}{\sqrt{2 \pi \sigma_{i}^{2}}} \exp -\frac{\left(\theta_{i}-f_{i}(\boldsymbol{X})\right)^{2}}{2 \sigma_{i}^{2}},
$$

where

$$
\boldsymbol{f}(\boldsymbol{X})=\left[\begin{array}{c}
\vdots \\
P_{0 i}-10 \gamma \log _{10} \frac{\left\|\boldsymbol{x}_{i}-\boldsymbol{a}_{j}\right\|}{d_{0}} \\
\vdots \\
P_{0 i}-10 \gamma \log _{10} \frac{\left\|\boldsymbol{x}_{i}-\boldsymbol{x}_{k}\right\|}{d_{0}} \\
\vdots \\
\arctan \left(\frac{x_{i \mathrm{y}}-a_{j \mathrm{y}}}{x_{i \mathrm{x}}-a_{j \mathrm{x}}}\right) \\
\vdots \\
\arccos \left(\frac{x_{i \mathrm{z}}-a_{j \mathrm{z}}}{\left\|\boldsymbol{x}_{i}-\boldsymbol{a}_{j}\right\|}\right) \\
\vdots
\end{array}\right], \boldsymbol{\sigma}=\left[\begin{array}{c}
\vdots \\
\sigma_{n_{i j}} \\
\vdots \\
\sigma_{n_{i k}} \\
\vdots \\
\sigma_{m_{i j}} \\
\vdots \\
\sigma_{v_{i j}} \\
\vdots
\end{array}\right]
$$

Maximizing the log of the likelihood function (5) with respect to $\boldsymbol{X}$ gives us

180

185

the ML estimate, $\hat{\boldsymbol{X}}$, of the unknown locations [44], as:

$$
\hat{\boldsymbol{X}}=\underset{\boldsymbol{X}}{\arg \min } \sum_{i=1}^{3\left|\mathcal{E}_{\mathcal{A}}\right|+\left|\mathcal{E}_{\mathcal{T}}\right|} \frac{1}{\sigma_{i}^{2}}\left[\theta_{i}-f_{i}(\boldsymbol{X})\right]^{2} .
$$

Asymptotically (for large data records) the ML estimator in (6) is the minimum variance unbiased estimator [44]. However, finding the ML estimate directly from (6) is not possible, since (6) is non-convex and has no closed-form solution. Nevertheless, in the remainder of this work we will show that the LS problem in (6) can be solved in a distributed manner by applying certain approximations. More precisely, we propose a convex relaxation technique leading to a distributed SOCP estimator that can be solved efficiently by interior-point algorithms [37], and a suboptimal estimator based on the GTRS framework leading to a distributed SR-WLS estimator, which can be solved exactly by a bisection procedure [38]. We also show that the proposed SOCP estimator can 
be generalized to solve the localization problem in (6) where, besides the target locations, their transmit powers are different and unknown.

\subsection{Assumptions}

We outline here some assumptions for the WSN (made for the sake of simplicity and without loss of generality):

(1) The network is connected and it does not change during the computation period;

(2) Measurement errors for RSS and AoA models are independent, and $\sigma_{n_{i j}}=$ $\sigma_{n}, \sigma_{m_{i j}}=\sigma_{m}$ and $\sigma_{v_{i j}}=\sigma_{v}, \forall(i, j) \in \mathcal{E}_{\mathcal{A}} \cup \mathcal{E}_{\mathcal{T}} ;$

(3) The necessary equipment for collecting the AoA measurements is installed at anchors exclusively;

(4) A coloring scheme of the network is available.

In assumption (1), we assume that the sensors are static and that there is no sensor/link failure during the computation period, and that there exists a path between any two sensors $i, j \in \mathcal{V}$. Assumption (2) is made for the sake of simplicity. Assumption (3) indicates that only anchors are suitably equipped to acquire the AoA measurements (e.g. with directional antenna or antenna array [20, 41, 42], or video cameras [43]), due to network costs. Finally, assumption (4) implies that a coloring scheme is available in order to color (number) the sensors and establish a working hierarchy in the network. More precisely, we assume that a second-order coloring scheme is employed, meaning that no sensor has the same color (number) as any of its one-hop neighbors nor its two-hop neighbors [45]-[47]. In this way, we avoid message collision and reduce the execution time of the algorithm, since sensors with the same color can work in parallel ${ }^{3}$.

\footnotetext{
${ }^{3}$ Note that the network coloring problem may be considered as an optimization problem where the goal is to minimize the number of different colors. Although interesting in its own right, we did not investigate this problem here, since it does not follow the main idea of our
} 


\section{Distributed Localization}

Notice that the problem in (6) is dependent on the locations and pairwise measurements between the adjacent sensors only. Thus, having the initial lo-

cation estimations of the targets, $\hat{\boldsymbol{X}}^{(0)}$, at hand, the problem in (6) can be divided, i.e., the minimization can be performed independently by each target using only the information gathered from its neighbors. Hence, rather than solving (6), which can be computationally exhausting (in large-scale WSNs), we break down (6) into sub-problems, which we solve locally (by each target) using iterative approach. Consequently, target $i$ updates its location estimate in each iteration, $t$, by solving the following local ML problem:

$$
\hat{\boldsymbol{x}}_{i}^{(t+1)}=\underset{\boldsymbol{x}_{i}}{\arg \min } \sum_{j=1}^{3\left|\mathcal{E}_{\mathcal{A}_{i}}\right|+\left|\mathcal{E}_{\mathcal{T}_{i}}\right|} \frac{1}{\sigma_{j}^{2}}\left[\theta_{j}-f_{j}\left(\boldsymbol{x}_{i}\right)\right]^{2}, \forall i \in \mathcal{T},
$$

where $\mathcal{E}_{\mathcal{A}_{i}}=\left\{j:(i, j) \in \mathcal{E}_{\mathcal{A}}\right\}$ and $\mathcal{E}_{\mathcal{T}_{i}}=\left\{k:(i, k) \in \mathcal{E}_{\mathcal{T}}, i \neq k\right\}$ represent the set of all anchor and all target neighbors of the target $i$ respectively, and the first $\left|\mathcal{E}_{\mathcal{A}_{i}}\right|+\left|\mathcal{E}_{\mathcal{T}_{i}}\right|$ elements of $f_{j}\left(\boldsymbol{x}_{i}\right)$ are given as:

$$
f_{j}\left(\boldsymbol{x}_{i}\right)=P_{0 i}-10 \gamma \log _{10} \frac{\left\|\boldsymbol{x}_{i}-\hat{\boldsymbol{a}}_{j}\right\|}{d_{0}}, \text { for } j=1, \ldots,\left|\mathcal{E}_{\mathcal{A}_{i}}\right|+\left|\mathcal{E}_{\mathcal{T}_{i}}\right|
$$

with

$$
\hat{\boldsymbol{a}}_{j}= \begin{cases}\boldsymbol{a}_{j}, & \text { if } j \in \mathcal{A}, \\ \hat{\boldsymbol{x}}_{j}^{(t)}, & \text { if } j \in \mathcal{T} .\end{cases}
$$

\subsection{Transmit Powers Are Known}

\subsubsection{Distributed SOCP Algorithm}

Assuming that $\hat{\boldsymbol{X}}^{(0)}$ is given, when the noise power is sufficiently small, from (1) we can write:

$$
\lambda_{i j}\left\|\boldsymbol{x}_{i}-\hat{\boldsymbol{a}}_{j}\right\| \approx d_{0}, \forall i \in \mathcal{T}, \forall j \in \mathcal{E}_{\mathcal{A}_{i}} \cup \mathcal{E}_{\mathcal{T}_{i}}
$$

work, and our algorithms do not depend on the applied scheme; it is mentioned here merely as an interesting fact that might prevent message retransmission in order to save energy in the network, and not as a fundamental part of our algorithms. 
where

$$
\lambda_{i j}= \begin{cases}10^{\frac{P_{i j}^{\mathcal{A}}-P_{0 i}}{10 \gamma}}, & \text { if } j \in \mathcal{A}, \\ 10^{\frac{P_{i j}^{\tau}-P_{0 i}}{10 \gamma}}, & \text { if } j \in \mathcal{T} .\end{cases}
$$

Similarly, from (3) and (4) we respectively get:

$$
\boldsymbol{c}_{i j}^{T}\left(\boldsymbol{x}_{i}-\boldsymbol{a}_{j}\right) \approx 0, \forall i \in \mathcal{T}, \forall j \in \mathcal{E}_{\mathcal{A}_{i}}
$$

and

$$
\boldsymbol{k}_{i j}^{T}\left(\boldsymbol{x}_{i}-\boldsymbol{a}_{j}\right) \approx\left\|\boldsymbol{x}_{i}-\boldsymbol{a}_{j}\right\| \cos \left(\alpha_{i j}^{\mathcal{A}}\right), \forall i \in \mathcal{T}, \forall j \in \mathcal{E}_{\mathcal{A}_{i}}
$$

where $\boldsymbol{c}_{i j}=\left[-\sin \left(\phi_{i j}^{\mathcal{A}}\right), \cos \left(\phi_{i j}^{\mathcal{A}}\right), 0\right]^{T}$ and $\boldsymbol{k}_{i j}=[0,0,1]^{T}$. According to the LS criterion and (8), (9) and (10) each target updates its location by solving the following problem:

$$
\begin{gathered}
\hat{\boldsymbol{x}}_{i}^{(t+1)}=\underset{\boldsymbol{x}_{i}}{\arg \min } \sum_{j \in \mathcal{E}_{\mathcal{A}_{i}} \cup \mathcal{E}_{\mathcal{T}_{i}}}\left(\lambda_{i j}\left\|\boldsymbol{x}_{i}-\hat{\boldsymbol{a}}_{j}\right\|-d_{0}\right)^{2} \\
+\sum_{j \in \mathcal{E}_{\mathcal{A}_{i}}}\left(\boldsymbol{c}_{i j}^{T}\left(\boldsymbol{x}_{i}-\boldsymbol{a}_{j}\right)\right)^{2}+\sum_{j \in \mathcal{E}_{\mathcal{A}_{i}}}\left(\boldsymbol{k}_{i j}^{T}\left(\boldsymbol{x}_{i}-\boldsymbol{a}_{j}\right)-\left\|\boldsymbol{x}_{i}-\boldsymbol{a}_{j}\right\| \cos \left(\alpha_{i j}^{\mathcal{A}}\right)\right)^{2} .
\end{gathered}
$$

The LS problem in (11) is non-convex and has no closed-form solution. To convert (11) into a convex problem, we introduce auxiliary variables $r_{i j}=$ $\left\|\boldsymbol{x}_{i}-\hat{\boldsymbol{a}}_{j}\right\|, \forall(i, j) \in \mathcal{E}_{\mathcal{A}} \cup \mathcal{E}_{\mathcal{T}}, \boldsymbol{z}=\left[z_{i j}\right], \boldsymbol{g}=\left[g_{i j}\right], \boldsymbol{p}=\left[p_{i j}\right]$, where $z_{i j}=$ $\lambda_{i j}^{\mathcal{A}} r_{i j}-d_{0}, \forall(i, j) \in \mathcal{E}_{\mathcal{A}} \cup \mathcal{E}_{\mathcal{T}}, g_{i j}=\boldsymbol{c}_{i j}^{T}\left(\boldsymbol{x}_{i}-\boldsymbol{a}_{j}\right)$, and $p_{i j}=\boldsymbol{k}_{i j}^{T}\left(\boldsymbol{x}_{i}-\boldsymbol{a}_{j}\right)-$ $r_{i j} \cos \left(\alpha_{i j}^{\mathcal{A}}\right), \forall(i, j) \in \mathcal{E}_{\mathcal{A}}$. We get:

$$
\underset{\boldsymbol{x}_{i}, \boldsymbol{r}, \boldsymbol{z}, \boldsymbol{g}, \boldsymbol{p}}{\operatorname{minimize}}\|\boldsymbol{z}\|^{2}+\|\boldsymbol{g}\|^{2}+\|\boldsymbol{p}\|^{2}
$$

subject to

$$
\begin{gathered}
r_{i j}=\left\|\boldsymbol{x}_{i}-\hat{\boldsymbol{a}}_{j}\right\|, \forall(i, j) \in \mathcal{E}_{\mathcal{A}} \cup \mathcal{E}_{\mathcal{T}}, \\
z_{i j}=\lambda_{i j} r_{i j}-d_{0}, \forall(i, j) \in \mathcal{E}_{\mathcal{A}} \cup \mathcal{E}_{\mathcal{T}}, \\
g_{i j}=\boldsymbol{c}_{i j}^{T}\left(\boldsymbol{x}_{i}-\boldsymbol{a}_{j}\right), \forall(i, j) \in \mathcal{E}_{\mathcal{A}}, \\
p_{i j}=\boldsymbol{k}_{i j}^{T}\left(\boldsymbol{x}_{i}-\boldsymbol{a}_{j}\right)-r_{i j} \cos \left(\alpha_{i j}^{\mathcal{A}}\right), \forall(i, j) \in \mathcal{E}_{\mathcal{A}} .
\end{gathered}
$$

Introduce epigraph variables $e_{1}, e_{2}$ and $e_{3}$, and apply second-order cone constraint relaxation of the form $\|\boldsymbol{z}\|^{2} \leq e_{1}$, to obtain:

$$
\underset{\boldsymbol{x}_{i}, \boldsymbol{r}, \boldsymbol{z}, \boldsymbol{g}, \boldsymbol{p}, e_{1}, e_{2}, e_{3}}{\operatorname{minimize}} e_{1}+e_{2}+e_{3}
$$


subject to

$$
\begin{aligned}
& \left\|\boldsymbol{x}_{i}-\hat{\boldsymbol{a}}_{j}\right\| \leq r_{i j}, \forall(i, j) \in \mathcal{E}_{\mathcal{A}} \cup \mathcal{E}_{\mathcal{T}}, \\
& z_{i j}=\lambda_{i j} r_{i j}-d_{0}, \forall(i, j) \in \mathcal{E}_{\mathcal{A}} \cup \mathcal{E}_{\mathcal{T}}, \\
& g_{i j}=\boldsymbol{c}_{i j}^{T}\left(\boldsymbol{x}_{i}-\boldsymbol{a}_{j}\right), \forall(i, j) \in \mathcal{E}_{\mathcal{A}}, \\
& \left\|\left[\begin{array}{c}
2 \boldsymbol{z} \\
e_{1}-1
\end{array}\right]\right\| \leq e_{1}+1, \|\left[\begin{array}{c}
p_{i j}=\boldsymbol{k}_{i j}^{T}\left(\boldsymbol{x}_{i}-\boldsymbol{a}_{j}\right)-r_{i j} \cos \left(\alpha_{i j}^{\mathcal{A}}\right), \forall(i, j) \in \mathcal{E}_{\mathcal{A}},\left\|\left[\begin{array}{c}
2 \boldsymbol{p} \\
e_{2}-1
\end{array}\right]\right\| \leq e_{2}+1,\left\|\left[\begin{array}{c}
e_{3}-1
\end{array}\right]\right\| \\
e_{3}+1 .
\end{array}\right.
\end{aligned}
$$

The problem in (13) is an SOCP problem, which can be efficiently solved by the CVX package [48] for specifying and solving convex programs. In the further text, we will refer to (13) as "SOCP".

\subsubsection{Distributed $S R-W L S$ Algorithm}

We can rewrite (8) as:

$$
\lambda_{i j}^{2}\left\|\boldsymbol{x}_{i}-\hat{\boldsymbol{a}}_{j}\right\|^{2} \approx d_{0}^{2}, \forall(i, j) \in \mathcal{E}_{\mathcal{A}} \cup \mathcal{E}_{\mathcal{T}}
$$

In order to give more importance to the nearby links, introduce weights, $\boldsymbol{w}=$ ${ }_{255}\left[\sqrt{w_{i j}}\right]$, where

$$
w_{i j}=1-\frac{\widehat{d}_{i j}}{\sum_{(i, j) \in \mathcal{E}_{\mathcal{A}} \cup \mathcal{E}_{\mathcal{T}}} \widehat{d}_{i j}} .
$$

In (10), substitute $\left\|\boldsymbol{x}_{i}-\hat{\boldsymbol{a}}_{j}\right\|$ with $\widehat{d}_{i j}$ described in (2). According to the WLS criterion and (14), (9) and (10) each target updates its location by solving the following problem:

$$
\begin{gathered}
\hat{\boldsymbol{x}}_{i}^{(t+1)}=\underset{\boldsymbol{x}_{i}}{\arg \min } \sum_{j \in \mathcal{E}_{\mathcal{A}_{i} \cup \mathcal{E}_{\mathcal{T}_{i}}}} w_{i j}\left(\lambda_{i j}^{2}\left\|\boldsymbol{x}_{i}-\hat{\boldsymbol{a}}_{j}\right\|^{2}-d_{0}^{2}\right)^{2} \\
+\sum_{j \in \mathcal{E}_{\mathcal{A}_{i}}} w_{i j}\left(\boldsymbol{c}_{i j}^{T}\left(\boldsymbol{x}_{i}-\boldsymbol{a}_{j}\right)\right)^{2} \\
+\sum_{j \in \mathcal{E}_{\mathcal{A}_{i}}} w_{i j}\left(\boldsymbol{k}_{i j}^{T}\left(\boldsymbol{x}_{i}-\boldsymbol{a}_{j}\right)-\widehat{d}_{i j} \cos \left(\alpha_{i j}^{\mathcal{A}}\right)\right)^{2} .
\end{gathered}
$$

The above WLS estimator is non-convex and has no closed-form solution. However, we can express (15) as a quadratic programming problem whose 
global solution can be computed efficiently [38]. Using the substitution $\boldsymbol{y}_{i}=$ $\left[\boldsymbol{x}_{i}^{T},\left\|\boldsymbol{x}_{i}\right\|^{2}\right]^{T}, \forall i \in \mathcal{T},(15)$ can be rewritten as:

$$
\hat{\boldsymbol{y}}_{i}^{(t+1)}=\underset{\boldsymbol{y}_{i}}{\arg \min }\left\|\boldsymbol{W}\left(\boldsymbol{A} \boldsymbol{y}_{i}-\boldsymbol{b}\right)\right\|^{2}
$$

subject to

$$
\boldsymbol{y}_{i}^{T} \boldsymbol{D} \boldsymbol{y}_{i}+2 \boldsymbol{l}^{T} \boldsymbol{y}_{i}=0
$$

where $\boldsymbol{W}=\boldsymbol{I}_{3} \otimes \operatorname{diag}(\boldsymbol{w})$,

265

$$
\boldsymbol{D}=\left[\begin{array}{cc}
\boldsymbol{I}_{3} & \mathbf{0}_{3 \times 1} \\
\mathbf{0}_{1 \times 3} & 0
\end{array}\right], \boldsymbol{l}=\left[\begin{array}{c}
\mathbf{0}_{3 \times 1} \\
-1 / 2
\end{array}\right],
$$$$
\text { i.e., } \boldsymbol{A} \in \mathbb{R}^{3\left|\mathcal{E}_{\mathcal{A}_{i}}\right|+\left|\mathcal{E}_{\mathcal{T}_{i}}\right| \times 4}, \boldsymbol{b} \in \mathbb{R}^{3\left|\mathcal{E}_{\mathcal{A}_{i}}\right|+\left|\mathcal{E}_{\mathcal{T}_{i}}\right| \times 1} \text {, and } \boldsymbol{W} \in \mathbb{R}^{3\left|\mathcal{E}_{\mathcal{A}_{i}}\right|+\left|\mathcal{E}_{\mathcal{T}_{i}}\right| \times 3\left|\mathcal{E}_{\mathcal{A}_{i}}\right|+\left|\mathcal{E}_{\mathcal{T}_{i}}\right|} \text {. }
$$

The objective function and the constraint in (16) are both quadratic. This type of problem is known as GTRS [38, 49], and it can be solved exactly by a bisection procedure [38]. We denote (16) as "SR-WLS" in the remaining text.

In summary, the derivation of the above approaches can be described in two parts. In the first part, the local non-convex ML estimator in (7) is approximated by a different non-convex estimator, (11) and (15) respectively. The use of the objective functions in (11) and (15) is motivated by the fact that we get a much smoother surface in comparison to (7), at a cost of introducing some bias 275 with respect to the ML solution (see Fig. 2). If the bias effect is small, we might reach the ML solution by employing a local search around the solution of (11) and (15). In the second part of our approach, we convert (11) and (15) into a convex problem and GTRS framework, by following the above procedures. 

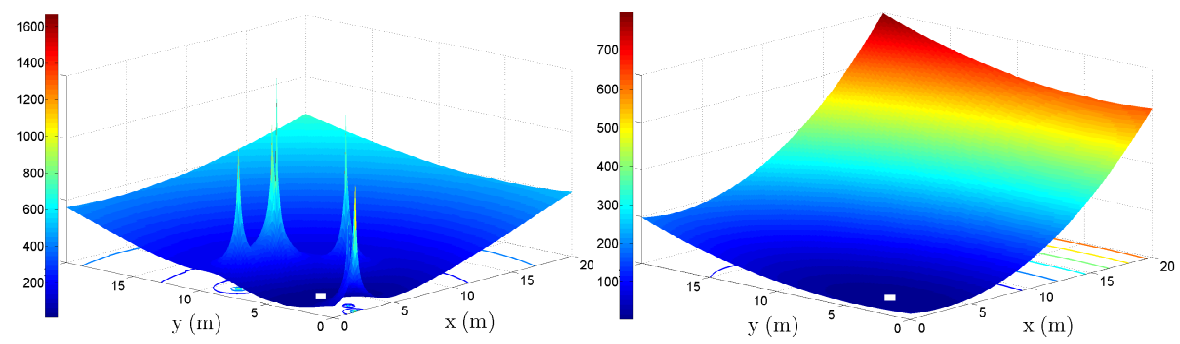

(a) Objective function in (7) using true sensors' locations

(b) Objective function in (11) after one it-

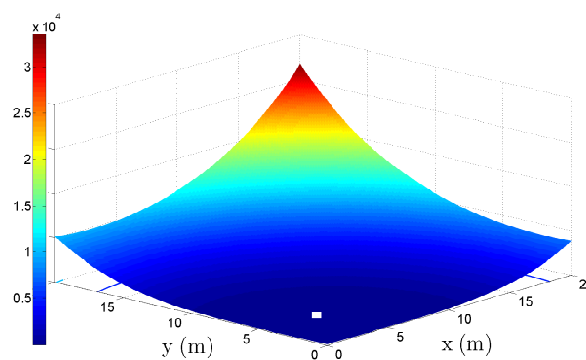
eration

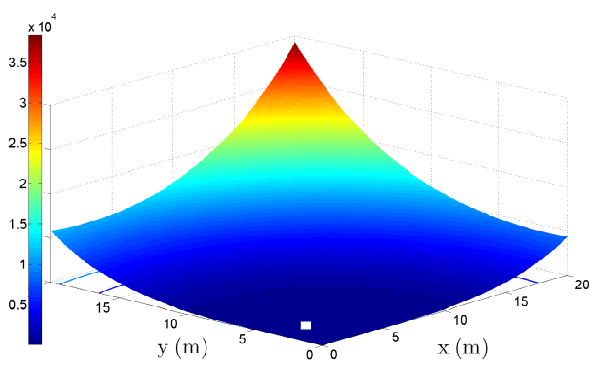

(c) Objective function in (15) after one itera- (d) Objective function in (15) after three itertion ations

Figure 2: Illustration of the objective functions in (7), (11) and (15) versus $x(\mathrm{~m})$ and $y(\mathrm{~m})$ coordinates (target location); the minimum of the objective function is indicated by a white square. 
Fig. 2 illustrates a realization of the objective function in (7), for the case

where the true sensors' locations were used and a realization of (11) and (15) after only one iteration, and (15) after three iterations, where the estimated targets' locations were used. The $i$-th target was located at [2.0;3.3], and it could directly communicate with its three anchor and three target neighbors. The noise standard deviation (STD) of RSS measurements was set to $\sigma_{n_{i j}}=2$ $\mathrm{dB}$ and the noise STD of angle measurements was set to $\sigma_{m_{i j}}=3 \mathrm{deg}$, and the rest of the parameters follow the set-up described in Section 5. On the one hand, in Fig. 2a, where the true sensors' locations were used, one can see that the objective function is highly non-convex and its global minimum is located at $[2.4 ; 3.5]$. Due to non-convexity of the problem, recursive algorithms, such as gradient search method, might get trapped into a local minimum, causing large error in the location estimation process. On the other hand, in Figs. 2b, 2c and $2 \mathrm{~d}$, where estimated targets' locations (obtained by solving the proposed "SOCP" and "SR-WLS" algorithm, respectively) were used, it can be seen that these objective functions are much smoother than the one in (7), and that the global minimum after only one iteration is located at $[2.5 ; 4.1]$ and $[4.3 ; 4.9]$ for (11) and (15), respectively and at $[2.4 ; 3.6]$ for (15) after three iterations. Because of the smoothness of the objective functions, the global minimum of the considered problems can be obtained uniquely and effortlessly for all targets via interior-point algorithms [37] and bisection procedure [38], by following the proposed procedures. However, the quality of the obtained solution will depend on the tightness of the performed relaxation. As we show in Section 5, the estimation accuracy betters as the number of iterations grows in general. Thus, we can conclude that the objective functions in (11) and (15) represent an excellent approximation of the original problem defined in (7).

Assuming that $\mathcal{C}$ represents the set of colors of the sensors, Algorithm 1 summarizes the proposed distributed SOCP and SR-WLS algorithms. Algorithm 1 is distributed in the sense that there is no central processor in the network, its coordination is carried out according to the applied coloring scheme, information exchange occurs between two incident sensors exclusively, and data 
most a broadcast of $3 \times T_{\max } \times M$ real values. Depending on which estimator is employed, in the remaining text, we label Algorithm 1 either as "SOCP" or as "SR-WLS".

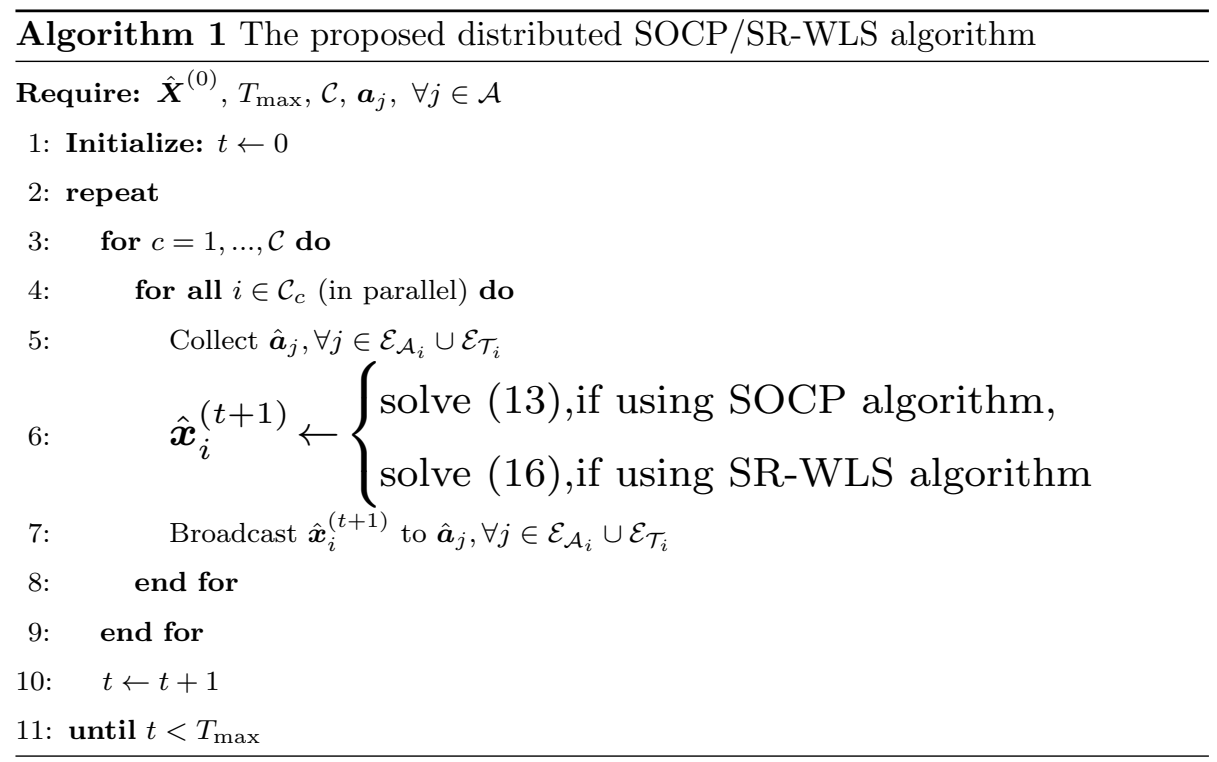

\subsection{Transmit Powers Are Not Known}

Often in practice testing and calibration are not the priority in order to restrict the implementation costs. Moreover, due to battery exhaust over time, sensors' transmit powers, $P_{i}$ 's, might change over time. Therefore, $P_{i}$ 's are often not calibrated, i.e., not known. Not knowing $P_{i}$ implies that $P_{0 i}$ is not known in the RSS model (1); see [10] and the references therein.

325

The generalization of the proposed SOCP estimator for known $P_{0 i}$ is straightforward for the case where $P_{0 i}$ is not known. More specifically, we can rewrite (8) 
as follows:

$$
\zeta_{i j}\left\|\boldsymbol{x}_{i}-\hat{\boldsymbol{a}}_{j}\right\| \approx \eta_{i} d_{0}, \forall i \in \mathcal{T}, \forall j \in \mathcal{E}_{\mathcal{A}} \cup \mathcal{E}_{\mathcal{T}},
$$

where $\eta_{i}=10^{\frac{P_{0 i}}{10 \gamma}}$ and

$$
\zeta_{i j}= \begin{cases}10^{\frac{P_{i j}^{\mathcal{A}}}{10 \gamma}}, & \text { if } j \in \mathcal{A}, \\ 10^{\frac{P_{i j}^{\top}}{10 \gamma}}, & \text { if } j \in \mathcal{T} .\end{cases}
$$

Following the LS concept and (17), (9) and (10), each target updates its

$$
\begin{gathered}
\left(\hat{\boldsymbol{x}}_{i}^{(t+1)}, \eta_{i}\right)=\underset{\boldsymbol{x}_{i}, \eta_{i}}{\arg \min } \sum_{j \in \mathcal{E}_{\mathcal{A}_{i} \cup \mathcal{E}_{\mathcal{T}_{i}}}}\left(\zeta_{i j}\left\|\boldsymbol{x}_{i}-\hat{\boldsymbol{a}}_{j}\right\|-\eta_{i} d_{0}\right)^{2} \\
+\sum_{j \in \mathcal{E}_{\mathcal{A}_{i}}}\left(\boldsymbol{c}_{i j}^{T}\left(\boldsymbol{x}_{i}-\boldsymbol{a}_{j}\right)\right)^{2}+\sum_{j \in \mathcal{E}_{\mathcal{A}_{i}}}\left(\boldsymbol{k}_{i j}^{T}\left(\boldsymbol{x}_{i}-\boldsymbol{a}_{j}\right)-\left\|\boldsymbol{x}_{i}-\boldsymbol{a}_{j}\right\| \cos \left(\alpha_{i j}^{\mathcal{A}}\right)\right)^{2} .
\end{gathered}
$$

By applying similar procedure as in Section 3.1.1, we obtain the following SOCP estimator:

$$
\underset{\boldsymbol{x}_{i}, \eta_{i}, \boldsymbol{r}, \boldsymbol{z}, \boldsymbol{g}, \boldsymbol{p}, e_{1}, e_{2}, e_{3}}{\operatorname{minimize}} e_{1}+e_{2}+e_{3}
$$

subject to

$$
\begin{aligned}
& \left\|\boldsymbol{x}_{i}-\hat{\boldsymbol{a}}_{j}\right\| \leq r_{i j}, \forall(i, j) \in \mathcal{E}_{\mathcal{A}} \cup \mathcal{E}_{\mathcal{T}}, \\
& z_{i j}=\zeta_{i j} r_{i j}-\eta_{i} d_{0}, \forall(i, j) \in \mathcal{E}_{\mathcal{A}} \cup \mathcal{E}_{\mathcal{T}} \text {, } \\
& g_{i j}=\boldsymbol{c}_{i j}^{T}\left(\boldsymbol{x}_{i}-\boldsymbol{a}_{j}\right), \forall(i, j) \in \mathcal{E}_{\mathcal{A}},
\end{aligned}
$$

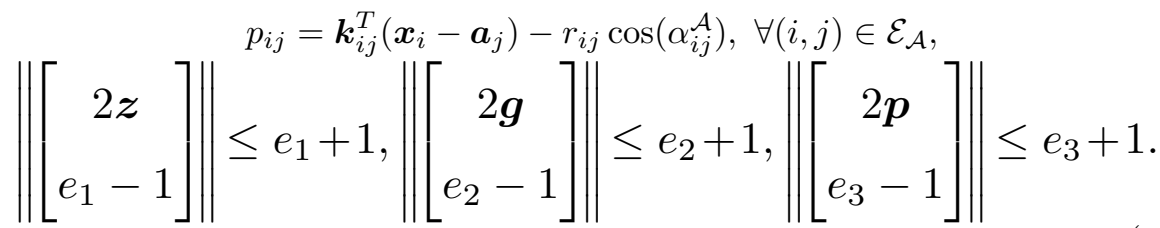

The problem in (19) is a classical SOCP, where the objective function and equality constraints are affine, and the inequality constraints are second-order cone constraints [37].

Algorithm 2 outlines the proposed SOCP algorithm for unknown $P_{i}$ 's. Lines 510 are performed concurrently by all targets $i \in \mathcal{C}_{c}$, which might reduce the running time of the algorithm. At Line 6 , we solve (19) $S$ number of times, after 

as if $P_{0 i}$ is known. Line 7 is introduced to avoid the oscillation in the location estimates. At Line 10, the location updates, $\hat{\boldsymbol{x}}_{i}^{(t+1)} \forall i \in \mathcal{T}$, are broadcasted to neighbors of $i$. In the remaining text, we label Algorithm 2 as "uSOCP".

\section{Complexity Analysis}

In order to evaluate the overall performance of a localization algorithm, it is necessary to analyze the trade off between the estimation accuracy and computational complexity. In this section, we investigate computational complexity of the considered algorithms. According to [50], the worst case computational complexity of an SOCP is:

$$
\mathcal{O}\left(\sqrt{L}\left(m^{2} \sum_{i=1}^{L} n_{i}+\sum_{i=1}^{L} n_{i}^{2}+m^{3}\right)\right)
$$

where $L$ is the number of the second-order cone constraints, $m$ is the number of the equality constraints, and $n_{i}$ is the dimension of the $i$-th second-order cone.

Assuming that $N_{\max }$ is the maximum number of steps in the bisection procedure, Table 1 provides a summary of the worst case computational complexities of the considered algorithms. In Table 1, the labels "SDP" and "uSOCP2" are used to denote the centralized SDP algorithm in [26] and the distributed SOCP algorithm in [27], respectively, which will be used later on in Section 5 to offer a better understanding of the performance of the proposed algorithms.

Table 1 shows that the computational complexity of a distributed algorithm depends mainly on the size of neighborhood fragments, rather than the total number of sensors in a WSN. Theoretically, it is possible to have a fully connected network, i.e., $\left|\mathcal{E}_{\mathcal{A}_{i}}\right|+\left|\mathcal{E}_{\mathcal{T}_{i}}\right|=M+N-1, \forall i \in \mathcal{T}$. However, in practice, the size of the neighborhood fragments are much smaller, due to energy restrictions (limited $R$ ). Therefore, distributed algorithms are a preferable solution in large-scale and highly-dense networks, since adding more sensors in the network will not have a severe impact on the size of neighborhood fragments. Table 1 also reveals that the proposed distributed SOCP algorithms are computationally 


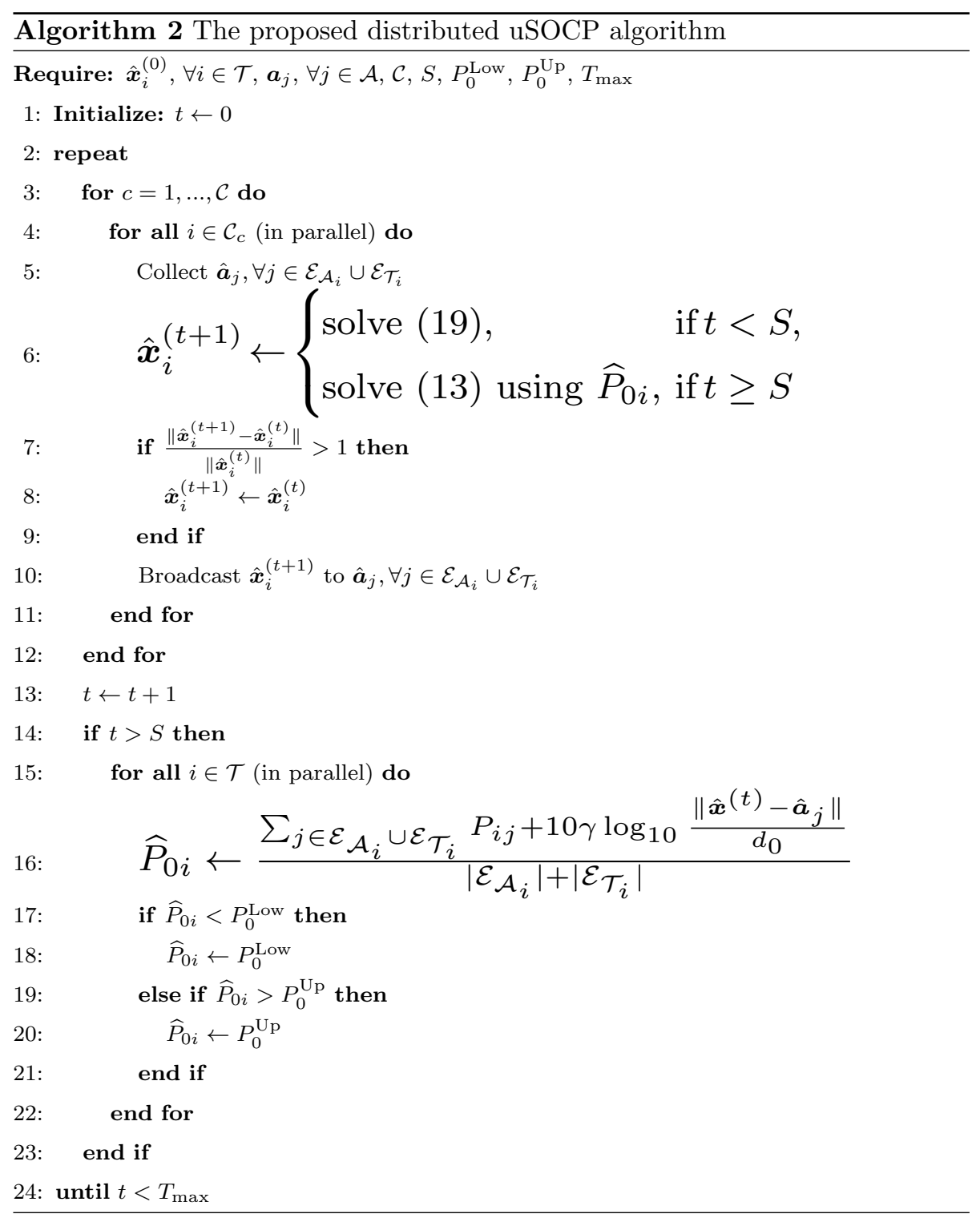


Table 1: Computational Complexity of the Considered Algorithms

\begin{tabular}{|c|c|}
\hline Algorithm & Complexity \\
\hline \hline SOCP & $T_{\max } \times M \times \mathcal{O}\left(\left(\max _{i}\left\{3\left|\mathcal{E}_{\mathcal{A}_{i}}\right|+\left|\mathcal{E}_{\mathcal{T}_{i}}\right|\right\}\right)^{3.5}\right)$ \\
\hline SR-WLS & $T_{\max } \times M \times \mathcal{O}\left(N_{\max } \times \max _{i}\left\{3\left|\mathcal{E}_{\mathcal{A}_{i}}\right|+\left|\mathcal{E}_{\mathcal{T}_{i}}\right|\right\}\right)$ \\
\hline $\mathrm{uSOCP}$ & $T_{\max } \times M \times \mathcal{O}\left(\left(\max _{i}\left\{3\left|\mathcal{E}_{\mathcal{A}_{i}}\right|+\left|\mathcal{E}_{\mathcal{T}_{i}}\right|\right\}\right)^{3.5}\right)$ \\
\hline $\mathrm{SDP}$ & $\mathcal{O}\left(\sqrt{3 M}\left(81 M^{4}\left(N+\frac{M}{2}\right)^{2}\right)\right)$ \\
\hline $\mathrm{uSOCP} 2$ & $T_{\max } \times M \times \mathcal{O}\left(\max _{i}\left\{\sqrt{3\left|\mathcal{E}_{\mathcal{A}_{i}}\right|+\left|\mathcal{E}_{\mathcal{T}_{i}}\right|}\right.\right.$ \\
& $\left.\left.\left(\left(3\left|\mathcal{E}_{\mathcal{A}_{i}}\right|\right)^{2}\left(3\left|\mathcal{E}_{\mathcal{A}_{i}}\right|+\mid \mathcal{E}_{\mathcal{T}_{i}}\right) \mid+\left(3\left|\mathcal{E}_{\mathcal{A}_{i}}\right|+\left|\mathcal{E}_{\mathcal{T}_{i}}\right|\right)^{2}\right)\right\}\right)$ \\
\hline
\end{tabular}

more demanding than the proposed SR-WLS one. This result is not surprising, since the SOCP approach employs sophisticated mathematical tools, whereas the SR-WLS approach applies the bisection procedure to solve the localization problem. Nevertheless, higher complexity of the proposed SOCP algorithms is justified by their superior performance in terms of the estimation accuracy and convergence, as we will see in Section 5 .

\section{Performance Results}

In this section, we present a set of results in order to asses the performance of the proposed approaches in terms of the estimation accuracy and convergence. All of the presented algorithms were solved by using the MATLAB package CVX [48], where the solver is SeDuMi [51]. In order to demonstrate the benefit of fusing two radio measurements versus traditional localization systems, we include also the performance results of the proposed methods when only RSS measurements are employed, called here "SOCP RSS" and "SR-WLS $_{\text {RSS". }}$ " To provide a performance benchmark, we employ also the existing distributed SOCP approach for unknown $P_{i}$ 's [27] labelled as "uSOCP2", as well as the centralized cooperative approach described in [26] for known $P_{i}$ 's which is used as a lower bound on the performance of the distributed approaches, denoted as "SDP". 
A random deployment of $M$ targets and $N$ anchors inside a cube region of length $B$ in each Monte Carlo $\left(M_{c}\right)$ run is considered. Random deployment of sensors is of particular interest, since the localization algorithms are tested against various network topologies in order to asses their robustness. In favor of making the comparison of the considered approaches as fair as possible, we first obtained $M_{c}=500$ targets' and anchors' locations, as well as noise realizations between two sensors $\forall(i, j) \in \mathcal{E}_{\mathcal{A}} \cup \mathcal{E}_{\mathcal{T}}, i \neq j$, in each $M_{c}$ run. Furthermore, we made sure that the network graph is connected in each $M_{c}$ run. We then solved the localization problem with the considered approaches for those scenarios. In all simulations presented here, the reference distance was set to $d_{0}=1 \mathrm{~m}$, the communication range of a sensor to $R=6.5 \mathrm{~m}$, the maximum number of steps in the bisection procedure to $N_{\max }=30$ and the PLE was fixed to $\gamma=3$. The true value of the reference power is drawn from a uniform distribution on an interval $\left[P_{0}^{\mathrm{Low}}, P_{0}^{\mathrm{Up}}\right]$, i.e., $P_{0 i} \in \mathcal{U}\left[P_{0}^{\mathrm{Low}}, P_{0}^{\mathrm{Up}}\right] \mathrm{dBm}$. Also, to account for a realistic measurement model mismatch and test the robustness of the new algorithms to imperfect knowledge of the PLE, the true PLE was drawn from $\gamma_{i j} \in \mathcal{U}[2.7,3.3], \forall(i, j) \in \mathcal{E}_{\mathcal{A}} \cup \mathcal{E}_{\mathcal{T}}, i \neq j$. Finally, we assumed that the initial guess of the targets' locations, $\hat{\boldsymbol{X}}^{(0)}$, is in the intersection of the big diagonals of the cube area.

The performance metric is the normalized root mean square error (NRMSE), defined as

$$
\mathrm{NRMSE}=\sqrt{\frac{1}{M M_{c}} \sum_{i=1}^{M_{c}} \sum_{j=1}^{M}\left\|\boldsymbol{x}_{i j}-\widehat{\boldsymbol{x}}_{i j}\right\|^{2}},
$$

where $\widehat{\boldsymbol{x}}_{i j}$ denotes the estimate of the true location of the $j$-th target, $\boldsymbol{x}_{i j}$, in the $i$-th Monte Carlo run.

Fig. 3 illustrates the NRMSE versus $t$ performance of the considered approaches when $N=20$ and $M=50$. From Fig. 3, we can see that the performance of all considered algorithms betters as $t$ grows, as anticipated. Furthermore, it can be noticed that the "uSOCP" curve gets saturated at $t=3$. Hence, at this point we start estimating $P_{0 i}$ 's, and continue our algorithm as if $P_{0 i}$ 's are known. This fact explains the sudden curve drop after $t=3$. One can argue 
that the proposed "uSOCP" algorithm shows excellent performance, outperforming noticeably the existing "uSOCP2" approach and achieving the lower bound provided by its counterpart for known $P_{i}$ 's. Also, it can be seen that the proposed hybrid methods outperform considerably their traditional counterparts that utilize RSS measurements only. Moreover, the "SR-WLS" method performs better than the "SOCP $\mathrm{RSS}$ " method in every iteration. This is important to note because the later method is computationally more demanding due to the use of sophisticated mathematical tools, which shows that even a simple algorithm such as the one based on bisection procedure can produce high estimation accuracy when two radio measurements are combined. One can perceive that all major changes in the performance for the considered algorithms take place in the first few iterations $(t \leq 10$ or $t \leq 20)$, and that the performance gain is negligible afterwards. This result is very important because it shows that our approaches require a low number of signal transmissions, which might enhance the utilization efficiency of the radio spectrum, a precious resource for wireless communications. It also shows that our algorithms are energy efficient; the communication phase is much more expensive (in terms of energy) than the data processing one [8]. Finally, the proposed SOCP performs outstanding, very close to the lower bound provided by the centralized "SDP" approach in just a few iterations.

Fig. 4 illustrates the NRMSE versus $t$ performance of the considered approaches when $N=30$ and $M=50$. Figs. 3 and 4 reveal that the performance 435 of all algorithms improves significantly as more anchors are added into the network. This behavior is expected, since when $N$ grows more reliable information and more AoA measurements are available in the network. Furthermore, Fig. 4 exhibits that the proposed hybrid algorithms outperform their RSS counterparts, and that they can be stopped after just $5-10$ iterations. Finally, although the new methods were derived under the assumption that the noise is small, we can see that they work excellent even when the assumption does not hold.

Fig. 5 illustrates the NRMSE versus $t$ performance of the considered ap- 


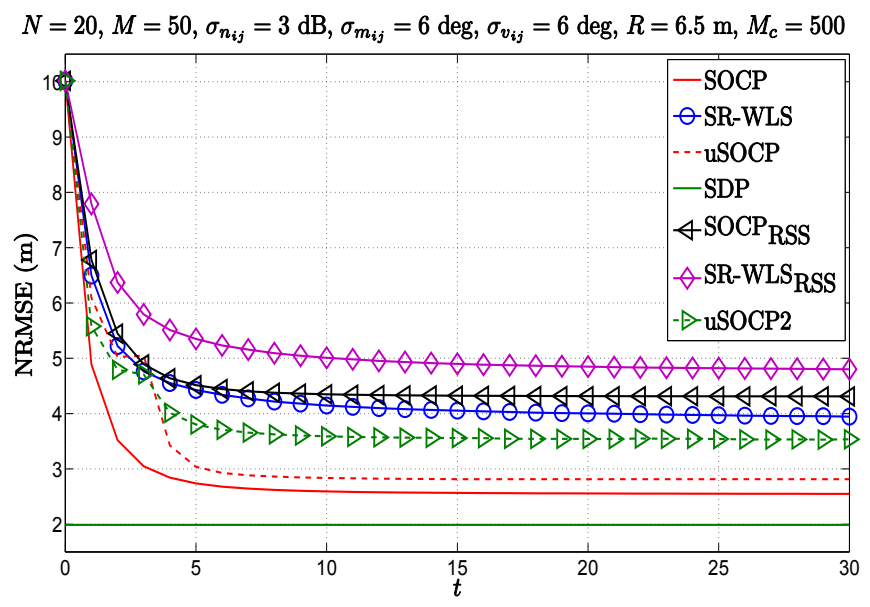

Figure 3: NRMSE versus $t$ comparison, when $N=20, M=50, R=6.5 \mathrm{~m}, \sigma_{n_{i j}}=3 \mathrm{~dB}$, $\sigma_{m_{i j}}=6 \mathrm{deg}, \sigma_{v_{i j}}=6 \mathrm{deg}, \gamma_{i j} \in \mathcal{U}[2.7,3.3], \gamma=3, B=20 \mathrm{~m}, P_{0 i} \in \mathcal{U}[-12,-8] \mathrm{dBm}$, $d_{0}=1 \mathrm{~m}, M_{c}=500$.

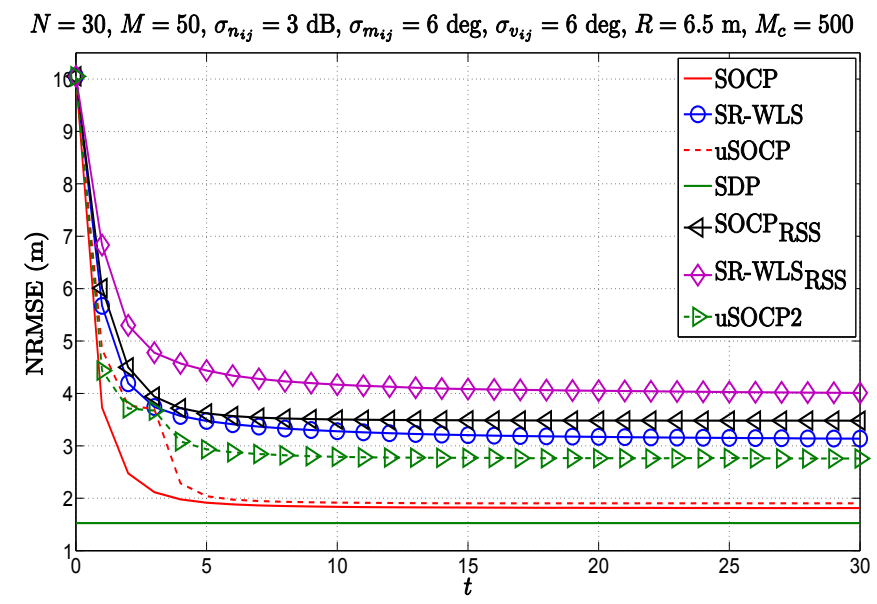

Figure 4: NRMSE versus $t$ comparison, when $N=30, M=50, R=6.5 \mathrm{~m}, \sigma_{n_{i j}}=3 \mathrm{~dB}$, $\sigma_{m_{i j}}=6 \mathrm{deg}, \sigma_{v_{i j}}=6 \mathrm{deg}, \gamma_{i j} \in \mathcal{U}[2.7,3.3], \gamma=3, B=20 \mathrm{~m}, P_{0 i} \in \mathcal{U}[-12,-8] \mathrm{dBm}$, $d_{0}=1 \mathrm{~m}, M_{c}=500$. 


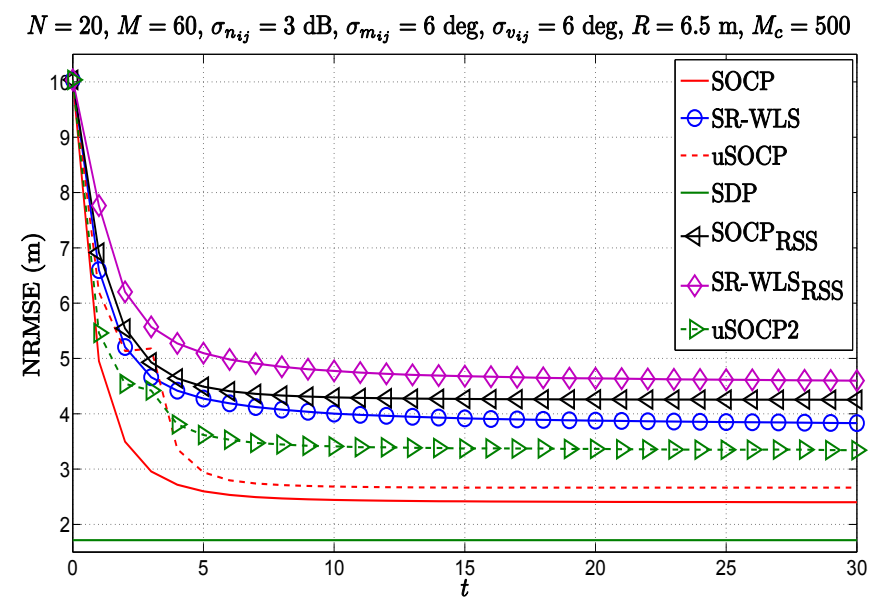

Figure 5: NRMSE versus $t$ comparison, when $N=20, M=60, R=6.5 \mathrm{~m}, \sigma_{n_{i j}}=3 \mathrm{~dB}$, $\sigma_{m_{i j}}=6 \mathrm{deg}, \sigma_{v_{i j}}=6 \mathrm{deg}, \gamma_{i j} \in \mathcal{U}[2.7,3.3], \gamma=3, B=20 \mathrm{~m}, P_{0 i} \in \mathcal{U}[-12,-8] \mathrm{dBm}$, $d_{0}=1 \mathrm{~m}, M_{c}=500$.

proaches when $N=20$ and $M=60$. From Figs. 3 and 5 it can be seen that the distributed approaches require a slightly higher number of iterations to converge when $M$ is increased. However, the estimation accuracy of the considered algorithms does not deteriorate when more targets are added in the network; it actually betters when $M$ is increased. Finally, Fig. 5 confirms the effectiveness of using the combined measurements in hybrid systems in comparison with using only a single measurement ${ }^{4}$.

In Figs. 6, 7 and 8 we investigate the impact of the quality of RSS and AoA measurements on the performance of the considered approaches. More precisely, Figs. 6, 7 and 8 respectively illustrate the NRMSE versus $\sigma_{n_{i j}}(\mathrm{~dB}), \sigma_{m_{i j}}(\mathrm{deg})$ and $\sigma_{v_{i j}}(\mathrm{deg})$ comparison, when $N=20, M=50, R=6.5 \mathrm{~m}$, and $T_{\max }=30$. In these figures, we can observe that the performance of all algorithms degrades as the quality of a certain measurement drops, as expected. It can also be seen that the quality of the RSS measurements has the most significant impact on

\footnotetext{
${ }^{4}$ Actually, in Figs. 3, 4 and 5 we have performed the simulations with $T_{\max }=200$ iterations in order to make sure that the considered approaches converge. In favour of a better overview, here, we present only the results for the first $t=30$ iterations.
} 


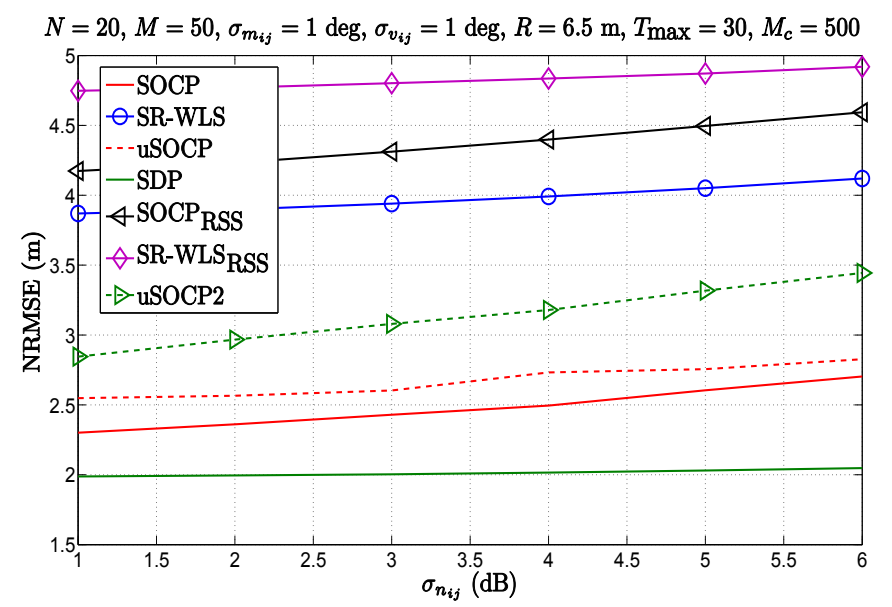

Figure 6: NRMSE versus $\sigma_{n_{i j}}(\mathrm{~dB})$ comparison, when $N=20, M=50, R=6.5 \mathrm{~m}, \sigma_{m_{i j}}=1$ $\mathrm{deg}, \sigma_{v_{i j}}=1 \mathrm{deg}, \gamma_{i j} \in \mathcal{U}[2.7,3.3], \gamma=3, T_{\max }=30, B=20 \mathrm{~m}, P_{0 i} \in \mathcal{U}[-12,-8] \mathrm{dBm}$, $d_{0}=1 \mathrm{~m}, M_{c}=500$.

the performance of the proposed algorithms, while the error in the azimuth and elevation angle measurements have marginal influence on the performance. This is not surprising, since the error of a few degrees in AoA measurements does not impair considerably their quality on a fairly short distance (communication range of all sensors is restricted to $R=6.5 \mathrm{~m}$ ), as shown in Figs. 7 and 8. On the other hand, RSS measurements are notoriously unpredictable [8]. Nonetheless, we can see from Fig. 6 that the performance loss is lower than $15 \%$ for the "SOCP" and "uSOCP", and 10\% for the "SR-WLS", which is relatively low for the considered error span. Finally, from the figures, we can see that the proposed "uSOCP" outperforms the existing "uSOCP2" for all settings.

\section{Conclusions}

In this work, we proposed two novel distributed algorithms to solve the RSS/AoA localization problem for known transmit powers based on SOCP relaxation technique and GTRS framework. The proposed SOCP algorithm provides exceptional localization accuracy in just a few iterations. Our algorithm based on GTRS framework is solved via a simple bisection procedure, and it 


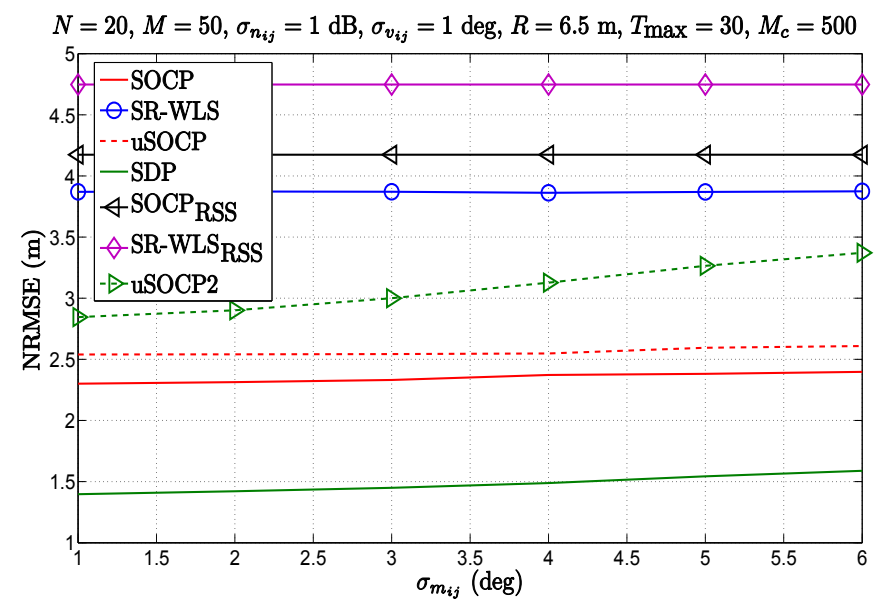

Figure 7: NRMSE versus $\sigma_{m_{i j}}(\mathrm{~dB})$ comparison, when $N=20, M=50, R=6.5 \mathrm{~m}, \sigma_{n_{i j}}=1$ $\mathrm{dB}, \sigma_{v_{i j}}=1 \mathrm{deg}, \gamma_{i j} \in \mathcal{U}[2.7,3.3], \gamma=3, T_{\max }=30, B=20 \mathrm{~m}, P_{0 i} \in \mathcal{U}[-12,-8] \mathrm{dBm}$, $d_{0}=1 \mathrm{~m}, M_{c}=500$.

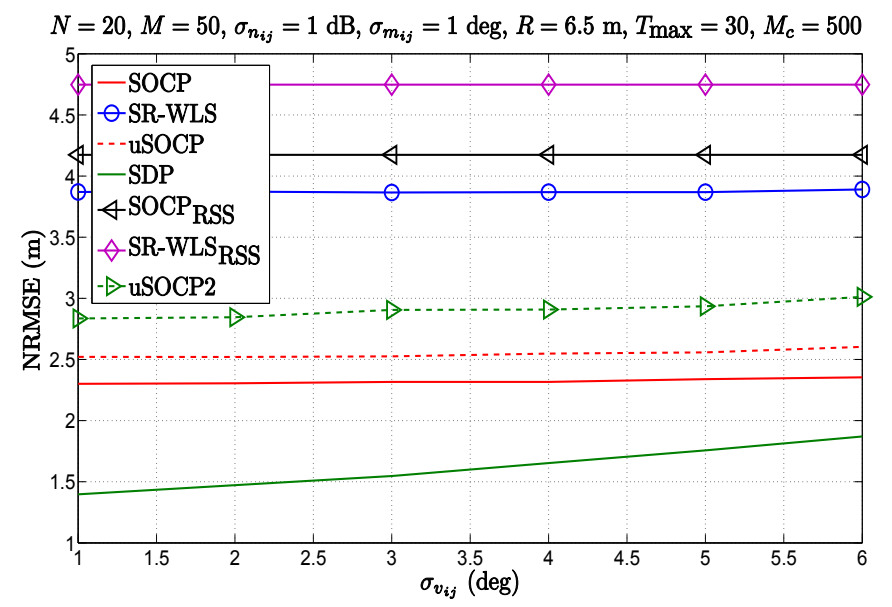

Figure 8: NRMSE versus $\sigma_{v_{i j}}(\mathrm{~dB})$ comparison, when $N=20, M=50, R=6.5 \mathrm{~m}, \sigma_{n_{i j}}=1$ $\mathrm{dB}, \sigma_{m_{i j}}=1 \mathrm{deg}, \gamma_{i j} \in \mathcal{U}[2.7,3.3], \gamma=3, T_{\max }=30, B=20 \mathrm{~m}, P_{0 i} \in \mathcal{U}[-12,-8] \mathrm{dBm}$, $d_{0}=1 \mathrm{~m}, M_{c}=500$. 
represents an excellent alternative to our SOCP algorithm, since its somewhat lower accuracy is compensated with linear computational complexity. We also show that the proposed SOCP algorithm for known transmit power can be generalized to the case where the transmit powers are different and not known. Our simulation results show that all of the proposed algorithms efficiently solve the very challenging cooperative localization problem, both in terms of the estimation accuracy and the convergence; the SOCP-based algorithm achieves the lower bound provided by the centralized SDP algorithm in only a few iterations, and outperforms notably the existing distributed approach. Furthermore, the simulation results confirmed the robustness of the proposed algorithms to the imperfect knowledge of the PLE, which is a very important practical scenario.

\section{${ }_{485}$ Appendix A. Second-order cone programming}

This appendix adds a supplementary explanation for second-order cone programming (SOCP). For more details see [32].

A general form of an SOCP problem is given as follows:

$$
\underset{\boldsymbol{x}}{\operatorname{minimize}} \boldsymbol{c}_{0}^{T} \boldsymbol{x}
$$

subject to

$$
\begin{aligned}
& \left\|\boldsymbol{A}_{i} \boldsymbol{x}+\boldsymbol{b}_{i}\right\| \leq \boldsymbol{c}_{i}^{T} \boldsymbol{x}+d_{i}(i=1, \ldots, m), \\
& \boldsymbol{F} \boldsymbol{x}=\boldsymbol{g}
\end{aligned}
$$

490

where $\boldsymbol{x} \in \mathbb{R}^{n}$ is the optimization variable, $\boldsymbol{A}_{i} \in \mathbb{R}^{n_{i} \times n}$, and $\boldsymbol{F} \in \mathbb{R}^{p \times n}$. A constraint of the form $\left\|\boldsymbol{A}_{i} \boldsymbol{x}+\boldsymbol{b}_{i}\right\| \leq \boldsymbol{c}_{i}^{T} \boldsymbol{x}+d_{i}$ is called a second-order cone constraint (SOCC). An SOCP problem is solvable in polynomial time by using interior point methods [32].

In addition, a hyperbolic constraint of the form $\|\boldsymbol{x}\|^{2} \leq y z$, for variables ${ }_{495} \boldsymbol{x} \in \mathbb{R}^{n}$ and $y, z \geq 0$, can be represented as an SOCC as below:

$$
\|\boldsymbol{x}\|^{2} \leq y z, \quad y, z \geq 0 \Longleftrightarrow\left\|\left[\begin{array}{c}
2 \boldsymbol{x} \\
y-z
\end{array}\right]\right\| \leq y+z .
$$




\section{Acknowledgement}

This work was partially supported by Fundação para a Ciência e a Tecnologia under Projects PEst-OE/EEI/UI0066/2014 (UNINOVA) and UID/EEA/50008/ 2013 (Instituto de Telecomunicações), project PTDC/EEI-TEL/6308/2014- HAMLeT, Program Investigador FCT (IF/00325/2015), as well as the grants SFRH/ BPD/108232/2015, SFRH/BD/91126/2012 and Ciência 2008 Post-Doctoral Research grant.

\section{References}

[1] Y. Singh, S. Saha, U. Chugh, and C. Gupta, "Distributed Event Detection in Wireless Sensor Networks for Forest Fires," UKSim, pp. 634-639, Apr. 2013.

[2] Z. Rongbai and C. Guohua, "Research on Major Hazard Installations Monitoring System Based on WSN," ICFCC, pp. V1-741-V1-745, May 2010.

[3] Z. Dai, S. Wang, and Z. Yan, "BSHM-WSN: A Wireless Sensor Network for Bridge Structure Health Monitoring," ICMIC, pp. 708-712, Jun. 2012.

[4] Lj. Blazevic, J. Y. Le Boudec, and S. Giordano, "A Location-based Routing Method for Mobile Ad Hoc Networks," IEEE Transactions on Mobile Computing, vol. 4, no. 2, pp. 97-110, Mar. 2005.

[5] L. Ghelardoni, A. Ghio, and D. Anguita, "Smart Underwater Wireless Sensor Networks," IEEEI, pp. 1-5, Nov. 2012.

[6] T. He, S. Krishnamurthy, J. A. Stankovic, T. Abdelzaher, L. Luo, R. Stoleru, T. Yan, and L. Gu, "Energy-Efficient Surveillance System Using Wireless Sensor Networks," MobiSys, pp. 1-14, Jun. 2004.

[7] B. K. Chalisea, Y. D. Zhanga, M. G. Amina, and B. Himed, "Target Localization in a Multi-static Passive Radar System Through Convex Optimization," Signal Process., vol. 102, pp. 207-215, Sep. 2014. 
[8] N. Patwari. Location Estimation in Sensor Networks. Ph.D. Thesis, University of Michigan, Ann Arbor, MI, USA, 2005.

[9] F. Bandiera, A. Coluccia, and G. Ricci, "A Cognitive Algorithm for Received Signal Strength Based Localization," IEEE Trans. Signal Process., vol. 63, no. 7, pp. 1726-1736, Apr. 2015.

[10] N. Patwari, J. N. Ash, S. Kyperountas, A. O. Hero III, R. L. Moses, and N. S. Correal, "Locating the Nodes: Cooperative Localization in Wireless Sensor Networks," IEEE Signal Processing Magazine, vol. 22, no. 4, pp. 5469, Jul. 2005.

[11] J. He, Y. Geng, and K. Pahlavan, "Toward Accurate Human Tracking: Modeling Time-of-Arrival for Wireless Wearable Sensors in Multipath Environment," IEEE Sensors J., vol. 14, no. 11, pp. 3996-4006, Nov. 2014.

[12] X. Qua and L. Xie, "An Efficient Convex Constrained Weighted Least Squares Source Localization Algorithm Based on TDOA Measurements," Signal Process., vol. 119, pp. 142-152, Feb. 2016.

[13] J. Cota-Ruiz, J. G. Rosiles, P. Rivas-Perea, and E. Sifuentes, "A Distributed Localization Algorithm for Wireless Sensor Networks Based on the Solutions of Spatially-Constrained Local Problems," IEEE Sensors J., vol. 13, no. 6, pp. 2181-2191, Jun. 2013.

[14] Y. Wang and K. C. Ho, "An Asymptotically Efficient Estimator in ClosedForm for 3D AOA Localization Using a Sensor Network," IEEE Trans. Wirel. Commun., vol. PP, no. 99, pp. 1, Jul.2015.

[15] S. Tomic, M. Beko, and R. Dinis, "RSS-based Localization in Wireless Sensor Networks Using Convex Relaxation: Noncooperative and Cooperative Schemes," IEEE Trans. Veh. Technol., vol. 64, no. 5, May 2015.

[16] N. Salman, M. Ghogho, and A. H. Kemp, "Optimized Low Complexity Sensor Node Positioning in Wireless Sensor Networks," IEEE Sensors J., vol. 14, no. 1, Jan. 2014. 
[17] A. Bahillo, S. Mazuelas, R. M. Lorenzo, P. Fernández, J. Prieto, R. J. Durán, and E. J. Abril, "Hybrid RSS-RTT Localization Scheme for Indoor Wireless Networks," EURASIP J. Advan. Sig. Process., vol. 2010, no. 1, pp. 1-12, Mar. 2010.

[18] U. Hatthasin, S. Thainimit, K. Vibhatavanij, N. Premasathian, and D. Worasawate, "The Use of RTOF and RSS for a One Base Station RFID system," IJCSNS, vol. 10, no. 7, pp. 862-865, Jul. 2010.

[19] T. Gädeke, J. Schmid, M. Krüger, J. Jany, W. Stork, and K. D. MüllerGlaser, "A Bi-Modal Ad-Hoc Localization Scheme for Wireless Networks Based on RSS and ToF Fusion," WPNC, pp. 1-6, Mar. 2013.

[20] K. Yu, "3-D Localization Error Analysis in Wireless Networks," IEEE Trans. Wirel. Commun., vol. 6, no. 10, pp. 3473-3481, Oct. 2007.

[21] S. Wang, B. R. Jackson, and R. Inkol, "Hybrid RSS/AOA Emitter Location Estimation Based on Least Squares and Maximum Likelihood Criteria," IEEE QBSC, pp. 24-29, Jun. 2012.

[22] L. Gazzah, L. Najjar, and H. Besbes, "Selective Hybrid RSS/AOA Weighting Algorithm for NLOS Intra Cell Localization," IEEE WCNC, pp. 25462551, Apr. 2014.

[23] Y. T. Chan, F. Chan, W. Read, B. R. Jackson, and B. H. Lee, "Hybrid Localization of an Emitter by Combining Angle-of-Arrival and Received Signal Strength Measurements," IEEE CCECE, pp. 1-5, May. 2014.

[24] S. Tomic, M. Marikj, M. Beko, R. Dinis and N.Órfão,, "Hybrid RSS-AoA Technique for 3-D Node Localization in Wireless Sensor Networks," IEEE IWCMC, pp. 1277-1282, Aug. 2015.

[25] C. Cheng, W. Hu, and W. P. Tay, "Localization of a moving noncooperative RF target in NLOS environment using RSS and AOA measurements," IEEE ICASSP, pp. 3581-3585, Apr. 2015. 
[26] S. Tomic, M. Beko and R. Dinis, "3-D Target Localization in Wireless Sensor Network Using RSS and AoA Measurement," accepted with minor revisions in the IEEE Trans. Vehic. Technol., Apr. 2016.

[34] G. Oliva, F. Pascucci, S. Panzieri, and R. Setola "Sensor Network Localization: Extending Trilateration via Shadow Edges," IEEE Trans. Autom. Control, vol. 60, no. 10, pp. 2752-2755, Oct. 2015. 
[35] A. Sahin, Y. S. Eroglu, I. Guvenc, N. Pala, and M. Yuksel, "Accuracy of AOA-based and RSS-based 3D Localization for Visible Light Communications," IEEE VTC pp. 1-5, Sep. 2015.

[36] R. Fletcher, Practical Methods of Optimization. John Wiley \& Sons, Chichester, UK, 1987.

[37] S. Boyd and L. Vanderberghe Convex Optimization. Cambridge University Press, Cambridge, UK, 2004.

[38] A. Beck, P. Stoica, and J. Li, "Exact and Approximate Solutions of Source Localization Problems," IEEE Trans. Sig. Process., vol. 56, no. 5, pp. 17701778, May 2008.

[39] T. S. Rappaport. Wireless Communications: Principles and Practice. Prentice-Hall: Upper Saddle River, NJ, USA, 1996.

[40] M. L. Sichitiu and V. Ramadurai, "Localization of Wireless Sensor Networks with a Mobile Beacon," IEEE MASS, pp. 174-183, Oct. 2004.

[41] Z. Xiang and U. Ozguner, "A 3-D Positioning System for Off-road Autonomous Vehicles," IEEE IV, pp. 130-134, Jun. 2005.

[42] B. de S. Muswieck, J. L. Russi, and M. V. T. Heckler, "Hybrid Method Uses RSS and AoA to Establish a Low-cost Localization System," SASE/CASE, pp. 1-6, Aug. 2013.

[43] M. B. Ferreira, J. Gomes, and J. P. Costeira, "A Unified Approach for Hybrid Source Localization Based on Ranges and Video," IEEE ICASSP, pp. 2879-2883, Apr. 2015.

[44] S. M. Kay. Fundamentals of Statistical Signal Processing: Estimation Theory. Prentice-Hall: Upper Saddle River, NJ, USA, 1993.

[45] S. C. Ergen and P. Varaiya, "TDMA Scheduling Algorithms for Wireless Sensor Networks", The Journal of Mobile Communication, Computation and Information, vol. 16, no. 4, pp. 985-997, May 2010. 
[46] J. F. C. Mota, J. M. F. Xavier, P. M. Q. Aguiar, and M. Püschel, "DADMM: A Communication-Efficient Distributed Algorithm for Separable Optimization", IEEE Trans. Signal Process., vol. 61, no. 10, pp. 2718-2723, May 2013.

[47] S. Tomic, M. Beko, and R. Dinis, "Distributed RSS-Based Localization in Wireless Sensor Networks Based on Second-Order Cone Programming," Sensors, vol. 14, no. 10, pp. 18410-18432, Oct. 2014.

[48] M. Grant and S. Boyd, CVX: Matlab Software for Disciplined Convex Programming. Version 1.21. Available online: http://cvxr.com/cvx (accessed on 15 April 2010).

[49] J. J. More, "Generalization of the Trust Region Problem," Optim. Meth. and Soft., vol. 2, pp. 189-209, 1993.

[50] I. Pólik and T. Terlaky, "Interior Point Methods for Nonlinear Optimization," in Nonlin. Optim., G. Di Pillo, F. Schoen, Eds. Springer, 1st Edition, 645 2010.

[51] J. F. Sturm, "Using SeDuMi 1.02, a MATLAB Toolbox for Optimization Over Symmetric Cones," Optim. Meth. Softw., 1998. 